\title{
45. APORTACIÓN A LA FLORA DE LAS SIERRAS DEL SUR DE ALBACETE (CALAR DEL MUNDO Y SIERRAS ADYACENTES)
}

\author{
Gemma LÓPEZ VÉLEZ
}

Palabras clave. Flora, fitosociología, Albacete, España.

Key words. Flora, fitosociology, Albacete, Spain.

Aportamos información sobre algunos taxones novedosos hallados en el SE de la provincia de Albacete, área favorecida por un enclave geográfico excepcional y punto de confluencia biogeográfica de cuatro subsectores, Alcaracense, Cazorlense, Subbético Murciano y Manchego Murciano. Comprende las sierras del Calar del Mundo, Padroncillo, Cujón, Ardal y núcleo sur del macizo de Alcaraz, con una altitud media de $1300 \mathrm{~m}$, donde el termótipo más extendido es el supramediterráneo subhúmedo-húmedo, con unas precipitaciones que a veces superan los $1000 \mathrm{~mm}$. Por debajo de esta cota, entre los 800 y 900 m se extiende el mesomediterráneo seco, llegando a ser semiárido en pequeños enclaves. A pesar del dominio de los afloramientos calizo-dolomíticos en las bases de estas sierras, existen otros silíceos pertenecientes a la facies Weald-Utrillas, lo que permite la presencia en el territorio de taxones que utilizan estas características edáficas y que son considerados como silicícolas. Todos ellos son de presencia poco común o rara. Los ejemplares se encuentran depositados en el Herbario MUB del Departamento de Biología Vegetal, Universidad de Murcia.

\section{Allium oleraceum L. var. oleraceum}

En Pastizales béticos supramediterráneos subhúmedos. El Pardal, 30SWH6260, 31.7.90, MUB 41830. Festucion scariosae. En la Península Ibérica alcanza el $\mathrm{N}$ y zonas elevadas del sur.

\section{Arum alpinum Schalt \& Kotschy}

En pastos de montaña o bajo encinares. Partes altas del Calar del Mundo, $1400 \mathrm{~m}, 30 \mathrm{SWH} 4753$, 17-6-84, MUB 41628. Supramediterráneo superior, subhúmedo. Elymo hispanici-Brachypodietum sylvaticix y Berberido hispanicae-Crataegetum laciniatae. Citada para Cazorla por Löve \& Kjellquist (1973) como Arum orientale subsp. lucanum y puesta en duda por Prime (1980) al afirmar que este taxón no alcanza la Península Ibérica. Los ejemplares recolectados coinciden completamente con la descripción que para Arum alpinum aporta Boyce (1993).

\section{Carex acutiformis Ehrh.}

Bajo saucedas en orillas muy encharcadas. Río de los Endrinales (Sierra de Alcaraz), $1400 \mathrm{~m}$, 30SWH5466, 27.9.91, MUB 34288 (Ríos et Alcaraz). Supramediterráneo superior. Salicetalia purpureae. En la Península Ibérica es abundante en el N, centro y centro-E.

Carex sylvatica Hudson subsp. sylvatica

En bosques caducifolios y setos. Lago de las Truchas, $1100 \mathrm{~m}$, 30SWH4659, 24.6.83, MUB 42621. Supramediterráneo. Querco-Fagetea. Medioeuropea. En la Península Ibérica abunda en el $\mathrm{N}$ y centro, es más rara hacia el SE.

\section{Centaurea alpina L.}

En pedregales calizos supramediterráneos del Calar de la Sima, 1700 m, 30SWH4543, 24.8.80, MUB 34356. Ombrotipo subhúmedo. LavanduloEchinospartium. Medioeuropea y mediterránea, se presenta muy puntualmente en el SE ibérico.

Centaurea nigra L. subsp. carpetana (Boiss. \& Reuter) Nyman

En prados umbrosos. Los Chorros, $1150 \mathrm{~m}$, 30SWH4956, 15.7.84, MUB 34395. Supramediterráneo subhúmedo a húmedo. Origanetalia vulgaris. 
Endemismo del W de los Pirineos y montañas del centro de la Península Ibérica. Es cita interesante para el enclave de Alcaraz y Albacete.

\section{Conopodium thalictrifolium (Boiss.) Calestani}

En grietas de lapiaz. Viboreros, $1690 \mathrm{~m} \mathrm{(Ca-}$ lar del Mundo), 30SWH5054, 6.6.82, MUB 40956. Supramediterráneo superior seco. Thlaspietea rotundifolii. Endémica del S y SE de la Península Ibérica.

\section{Cuscuta planiflora Ten}

Sobre suelos básicos y huespedes variados. Cañada de los Mojones, $1350 \mathrm{~m}$ (Calar del Mundo), 30SWH4856, 3.6.84, MUB 41127. En la Península Ibérica en zonas altas del S (Béticas, Sierra Nevada).

\section{Cynara baetica (Sprengel) Pau}

Muy ligado, al menos en los subsectores Cazorlense y Alcaracense, a los afloramientos triásicos (Keuper). Fábricas de Resinas, $900 \mathrm{~m}$, 30SWH4053, 18.8.79, MUB 34680. Mesomediterráneo superior a supramediterráneo, subhúmedo. Onopordion nervosi. Endemismo del S de España.

\section{Erodium glandulosum (Cav.) Willd.}

Muy local en el SW del territorio de estudio, en roquedos y rellanos rocosos de Los Voladores (Calar de la Sima), 1700 m, 30SWH4644, 25.5.81, MUB 41091). Supramediterráneo superior seco. Potentilletalia. Endémica del N de España, penetra en forma de cuña en localidades de Castilla la Vieja (Burgos) y Castilla la Nueva (Cuenca). Es una importante cita para la provincia, donde podría tener su límite meridional.

\section{Erigeron alpinus L.}

En prados de sustrato rocoso. Sierra del Ardal, $1400 \mathrm{~m}, 30$ SWH5646, 15.3.80, MUB 10333). Supramediterráneo superior seco. En la Península Ibérica, en Pirineo Central.

Euphorbia baetica Boiss.

Sobre sustrato arenoso de la Facies Utrillas, que sustentan notable influencia luso-extremadurense. Sierra del Ardal, 1250 m, 30SWH5646, 20.10.80, MUB 40497. Hyacinthoido-Quercetum cocciferae. Endemismo del SW de la Península Ibérica.

Jasione montana L. subsp. blepharodon (Boiss. et Reuter) Rivas Martínez
En prados terofíticos sobre sustratos pedregosos. Calar de la Sima, 1560 m, 30SWH4543, 9.6.80, MUB 41436. Supramediterráneo superior seco. Tuberarietea guttatae. La subespecie blepharodon se distribuye por el SW de la Península y por las sierras béticas.

\section{Leucanthemum discoideum (All.) Coste}

Ligada a zonas de laderas pedregosas y márgenes de bosque que rodean el Calar del Mundo, $1100 \mathrm{~m}, 30 \mathrm{SWH} 4657,15.6 .80$, MUB 9079. Mesomediterráneo a supramediterráneo subhúmedo. Lavandulo-Echinospartion. Area de distribución desde el SE de Francia (Alpes Marítimos, Niza) y SW de Italia. En la Península Ibérica en enclaves montanos del NE, E y SE (Sierras de Segura y S de Albacete). Vojt (1991) considera que el taxón segurense corresponde a L. aligulatum y que carece de parentesco con $L$. discoideum. El material del área de estudio muestra una variabilidad morfológica mayor que la que se supone para $L$. aligulatum, observándose notables solapamientos con lo que Vojt considera caracteres propios de $L$. discoideum, por lo que pensamos que no son tan claras las diferencias y mantenemos el sentido clásico del taxón

Linaria amethystea (Vent.) Hoffmanns et Link subsp. multipuctata (Brot) Chater \& D.A. Webb.

Escasa y puntual. Con preferencia en terrenos neutros o descalcificados, formando prados de terófitos, Calar del Mundo, 1350 m, 30SWH4856, 21.5.80, MUB 41286. Supramediterráneo. Tuberarietalia guttatae. Endémica del centro de Portugal y SW de España.

Luzula campestris (L.) DC. in Lam. et DC. subsp. nevadensis Monserrat

En prados de montaña con humedad edáfica. Laguna del Pocico (Calar del Mundo), $1470 \mathrm{~m}$, 30SWH4447, 29.5.93, MUB 41718. Supramediterráneo. Molinio-Arrhenatheretea. S de la Península Ibérica.

Luzula fosteri (Sm.) DC. in Lam. et DC. subsp. baetica Monserrat

En bosques caducifolios de zonas mesomediterráneas a supramediterráneas subhúmedas. Lago de las Truchas (Valle de los Chorros), 1100 $\mathrm{m}, \quad 30$ SWH4957, 25.4.84, MUB 41722. 
Origanetalia. Endémica del SW de la Península Ibérica, podría tener aquí su límite meridional.

Onobrychis humilis (Loefl.) G. López subsp. matritensis (Boiss. \& Reuter) Greuter \& Burdet Muy abundante. En pastos secos sobre terrenos margosos. Riópar, $1150 \mathrm{~m}, 30 \mathrm{SWH} 5161$, 17.5.81, MUB 32665. Mesomediterráneo a supramediterráneo, seco. Helichryso-Santolinetalia. Elemento Ibero-magrebí. Distribuido en áreas reducidas en centro, SE y E peninsular.

\section{Ranunculus lateriflorus DC.}

Bordes de lagunas que se desecan o reducen al final de la primavera o verano. Sobre sustratos descalcificados. Laguna del Pocico (Calar del Mundo), $1470 \mathrm{~m}, 30 \mathrm{SWH} 4447,29.5 .90$, MUB 41645. Supramediterráneo superior. Isoeto-Nanojuncetea. En la Península Ibérica centro y centro-E. Más rara hacia el SE.

\section{Ranunculus malessanus Degen le Hervier}

En partes cacuminales del Calar del Mundo, $1600 \mathrm{~m}$ y Calar de la Sima, $1800 \mathrm{~m}, 30$ SWH4543, 25.5.81, MUB 30385. Supramediterráneo. MolinioArrhenatheretea. Endémica de las sierras de Cazorla y Segura.

\section{Scirpus lacustris L.}

En aguas de «uvalas» que se reducen en verano. Laguna del Cortijo del Pocico (Calar del Mundo), $1470 \mathrm{~m}, 30$ SWH4348, 1.5.80, MUB 41340. Supramediterráneo superior. Holoschoenetalia. En la Península Ibérica N, centro, SW y Sierras de Cazorla y Segura.

Succisella andreae-molinae Escudero \& Pajarón En nuestra área sobre sustratos silíceos de la facies Utrillas con abundante humedad edáfica. Arroyo del Padroncillo, 1100 m, 30SWH4659, Sept.91, MUB 5659. Mesones, 950 m, 30SWH5660, 11.7.79, MUB 41666. Mesomediterráneo subhúmedo. Holoschoenetalia. Endemismo de las Sierras de Segura y Alcaraz.

Thalictrum foetidum L. subsp. valentinum $O$. Bolós \& Vigo

En gleras supramediterráneas del Calar de la Sima, 1800 m, 30SWH4643, 16.6.91, MUB 40813. Thlaspietea rotundifolii. Endemismo del E peninsular.

\section{Verbascum hervieri Degen}

Herbazales de zonas nitrificadas en margas del Keuper. Arroyo de la Puerta, $1170 \mathrm{~m}$, 30SWH4654, 16.6.83, MUB 41342. Mesomediterráneo subhúmedo. Onopordenea acanthii. Endémica del SE de España.

\section{BIBLIOGRAFÍA}

BOYCE, P. -1993- The Genus Arum. The Royal Botanic Gardens, Kew, London, Magacine Monograph: 196.

LÓVE, A. y E. KJELLQUIST. - 1973- Cytotaxonomy of spanish plants II. Monocotyledons. Lagascalia, 3(2): 147-182.

PRIME, C.T. -1980- Arum L. in: Tutin et al. (eds.) Flora Europea 5: 269-271. Cambridge.

VOGT, R. -1991- Die Gattung Leucanthemum Mill. (Compositae-Anthemidae) auf der Iberischen Halbinsel. Ruizia, 10: 261.

Aceptado para su publicación en Junio de 1994

Dirección de la autora. Departamento de Biología Vegetal, Facultad de Biología, Universidad de Murcia, Campus de Espinardo, 30100-Murcia. 